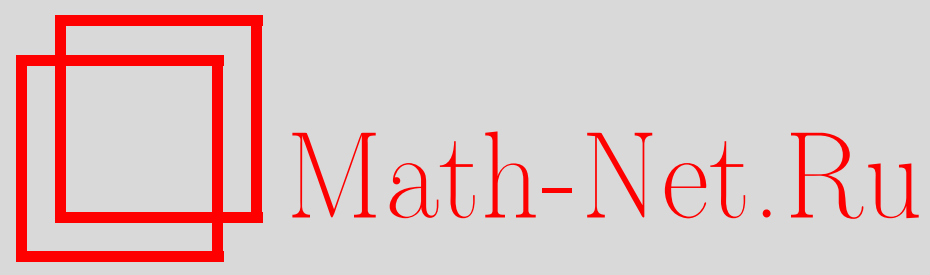

K. В. Панкрашкин, Локальность квадратичных форм для точечных возмущений операторов Шредингера, Матем. заметки, 2001, том 70, выпуск 3, 425-433

DOI: https://doi.org/10.4213/mzm754

Использование Общероссийского математического портала Math-Net.Ru подразумевает, что вы прочитали и согласны с пользовательским соглашением http://www.mathnet.ru/rus/agreement

Параметры загрузки:

IP : 54.237 .59 .107

26 апреля 2023 г., $14: 35: 16$

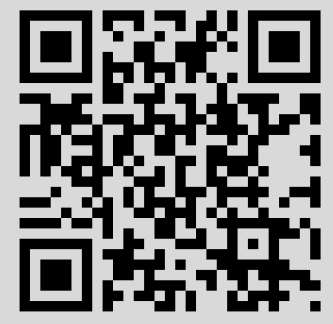




\section{ЛОКАЛЬНОСТЬ КВАДРАТИЧНЫХ ФОРМ ДЛЯ ТОЧЕЧНЫХ ВОЗМУШЕНИЙ ОПЕРАТОРОВ ШРЕДИНГЕРА}

\section{К. В. Панкрашкин}

В работе изучаются точечные возмущения операторов Шредингера в контексте теории самосопряженных расширений Крейна. Доказан критерий локальности квадратичной формы для таких возмущений.

Библиография: 16 названий.

Введение. Точечными возмущениями принято назьвать операторы, формально имеюшие вид

$$
H=H^{0}+\sum_{\mathbf{a} \in A} \lambda^{\mathbf{a}} \delta(\cdot-\mathbf{a}),
$$

где $H^{0}$ - самосопряженньй оператор в $L^{2}\left(\mathbb{R}^{\nu}\right), A$ - дискретное подмножество $\mathbb{R}^{\nu}, \delta$ дельта-функция Дирака. Строгий математический смысл выражению (1) можно придать с использованием теории самосопряженных расширений, при этом возникает и соответствующая параметризация точечных возмущений [1], [2]. Однако семейство возникающих при этом операторов довольно обширно и включает в себя операторы с весьма экзотическими свойствами. Пусть, например, $H^{0}$ - оператор Ландау (в частности, оператор Лапласа). Возмущения такого $H^{0}$ регулярными периодическими потенциалами в так называемом случае рационального потока имеют зонньй спектр [3]. Желательно было бы сохранить регулярную структуру спектра и при замене регулярного потенциала точечньм. Однако дело обстоит иначе, а именно: cyществуют периодические точечные возмущения $H^{0}$, имеющие в своем спектре участки с фрактальной структурой [4], [5]. Тем не менее, появления подобной патологии можно избежать, ограничившись рассмотрением точечных возмущений, обладающих свойством форм-локальности [5]. Напомним, что оператор $B$ в $L^{2}(X, d \mu)$, имеющий квадратичную форму $b$ с областью определения $\mathbf{D}(b)$, предложено назьвать форм-локальным или локальным в смысле форм, если $b(\varphi, \psi)=0$ для любых $\varphi, \psi$ из $\mathbf{D}(b)$ таких, что $\operatorname{supp} \varphi \cap \operatorname{supp} \psi=\varnothing$ (квадратичная форма $b$ при этом называется локальной) [6]. Заметим, что обычное определение локальности (оператор $B$, действующий в $L^{2}(X, d \mu)$, принято называть локальным, если для любой функции $\varphi$ из его области определения

Работа выполнена при поддержке DFG-PAH, грант № 436 RUS113/572. 
выполнено $\operatorname{supp} B \varphi \subset \operatorname{supp} \varphi)$ не является различающим в данном случае, поскольку все точечные возмущения локального оператора также локальны [1].

Понятие форм-локальности представляет интерес также и с точки зрения подхода к определению точечного возмушения (1) как предела операторов вида

$$
H_{n}=H^{0}+\sum_{\mathbf{a} \in A} \lambda_{n}^{\mathbf{a}} f_{n}(\cdot-\mathbf{a}), \quad n \rightarrow \infty
$$

при $f_{n} \rightarrow \delta$. Уже в случае, когда $H^{0}=-\Delta$ и множество $A$ конечно, такой подход применим лиш к точечным возмущениям, соответствующим так назьваемой модели независимых центров [1]. В то же время в [6] замечено, что только такие точечные возмущения и являются локальными в смысле форм.

В настоящей работе решается следующая задача: среди точечных возмущений оператора Шредингера выделить те, которые являются форм-локальньми. Основным результатом работы является теорема 2 , дающая необходимое и достаточное условие локальности в смысле форм для точечных возмущений двух- и трехмерных операторов Шредингера.

1. Точечные возмущения и формула Крейна. Мы будем использовать следующие обозначения: $\mathbf{D}(B), \mathbf{Q}(B), \rho(B), \sigma(B)$ - область определения, область определения квадратичной формы, резольвентное множество и спектр оператора $B$ соответственно; $\mathscr{L}(\mathscr{G}, \mathscr{H})$ - пространство ограниченных операторов из $\mathscr{G}$ в $\mathscr{H}, \mathcal{S}(\mathscr{G})$ - пространство самосопряженных операторов в $\mathscr{G}$. Скалярное произведение будем считать линейным по второму аргументу.

Введем теперь строгое определение точечного возмущения для оператора Шредингера.

Пусть $\mathbf{a}_{1}, \ldots, \mathbf{a}_{m}$ - линейно независимые векторы из $\mathbb{R}^{\nu}, m \leqslant \nu \leqslant 3$,

$$
\Lambda=\left\{\sum_{i=1}^{m} k_{i} \mathbf{a}_{i}: k_{i} \in \mathbb{Z}\right\}
$$

$K$ - произвольное конечное подмножество $\mathbb{R}^{\nu}$ и $A=\Lambda+K$ (мы не исключаем случай $m=0$, т.е. $\Lambda=\{0\}, A=K)$.

Пусть также $\mathbf{A}: \mathbb{R}^{\nu} \rightarrow \mathbb{R}^{\nu}$ - дважды непрерывно дифференцируемая вектор-функция, $V: \mathbb{R}^{\nu} \rightarrow \mathbb{R}$ - гладкая полуограниченная снизу функция и, кроме того, $\mathbf{A}$ и $V$ периодичны относительно $\Lambda$, т.е. выполнены следующие условия:

$$
\mathbf{A}\left(\mathbf{x}+\mathbf{a}_{i}\right) \equiv \mathbf{A}(\mathbf{x}), \quad V\left(\mathbf{x}+\mathbf{a}_{i}\right) \equiv V(\mathbf{x}), \quad i \leqslant m .
$$

Через $H^{0}$ обозначим оператор Шредингера с векторньм потенциалом магнитного поля $\mathbf{A}$ и электрическим потенциалом $V$ :

$$
H^{0}=(i \nabla+\mathbf{A})^{2}+V .
$$


Известно, что выполнены включения [7]

$$
\begin{aligned}
& \mathbf{D}\left(H^{0}\right) \subset C\left(\mathbb{R}^{\nu}\right) \\
& \mathbf{Q}\left(H^{0}\right) \subset H_{\mathrm{loc}}^{1}\left(\mathbb{R}^{\nu}\right)
\end{aligned}
$$

В силу первого из этих включений корректно определены множество $\mathbf{D}(S)$ и оператор $S$ :

$$
\mathbf{D}(S)=\left\{\varphi \in \mathbf{D}\left(H^{0}\right): \varphi(\mathbf{a})=0 \quad \forall \mathbf{a} \in A\right\}, \quad S=\left.H^{0}\right|_{\mathbf{D}(S)}
$$

Точечным возмущением оператора $H^{0}$, сосредоточенньп на $A$, называют любое самосопряженное расширение $H$ оператора $S$, дизъюнктное с $H^{0}$, т.е. такое, что $\mathbf{D}(H) \cap$ $\mathbf{D}\left(H^{0}\right)=\mathbf{D}(S)$. Удобное описание точечных возмущений дает теория самосопряженных расширений Крейна [8], основные положения которой мы сейчас напомним.

Пусть $\mathscr{H}$ - гильбертово пространство, $S$ - замкнутый симметрический оператор в $\mathscr{H}$ с индексами дефекта $(n, n), 0<n \leqslant \infty, H^{0}$ - фиксированное самосопряженное расширение $S$. Зафиксируем гильбертово пространство $\mathscr{G}$ такое, что $\operatorname{dim} \mathscr{G}=n$. Отображение $z \mapsto \Gamma_{z}$ из $\rho\left(H^{0}\right)$ в $\mathscr{L}(\mathscr{G}, \mathscr{H})$ назьвается Г-полем пары $\left(S, H^{0}\right)$, если вьполнены следующие условия:

I) $\Gamma_{z}$ - линейно-топологический изоморфизм $\mathscr{G}$ на $\operatorname{Ker}\left(S^{*}-z\right)$;

II) для всех $z, \zeta \in \rho\left(H^{0}\right)$ вьполнено равенство $\Gamma_{z}=\left(H^{0}-\zeta\right)\left(H^{0}-z\right)^{-1} \Gamma_{\zeta}$.

Оператор-функция $Q(z)$ на $\rho\left(H^{0}\right)$ со значениями в $\mathscr{L}(\mathscr{G}, \mathscr{G})$ назьвается $\mathscr{Q}$-функцией пары $\left(S, H^{0}\right)$, если она обладает свойством $Q(\zeta)-Q(z)^{*}=(\zeta-\bar{z}) \Gamma_{z}^{*} \Gamma_{\zeta}$. Функция $Q(z)$ определена с точностью до слагаемого $C \in \mathscr{L}(\mathscr{G}, \mathscr{G}) \cap \mathcal{S}(\mathscr{G})$, выбрав которое можно положить

$$
Q(z)=C-i \operatorname{Im} \zeta \Gamma_{\zeta}^{*} \Gamma_{\zeta}+(z-\bar{\zeta}) \Gamma_{\zeta}^{*} \Gamma_{z}
$$

где $\zeta$ - фиксированный элемент из $\rho\left(H^{0}\right)$.

ТЕорема 1 (формула Крейна). Выражение

$$
R(z)=R^{0}(z)-\Gamma_{z}(Q(z)-T)^{-1} \Gamma_{\bar{z}}^{*}
$$

әде $R^{0}(z)=\left(H^{0}-z\right)^{-1}-$ резольвента оператора $H^{0}$, устанавливает взаимно однозначное соответствие между самосопряженными операторами $T$, действующими в $\mathscr{G}$, и резольвентами $R(z)$ самосопряженных расширений оператора $S$, дизбюнктными с $H^{0}$.

В дальнейшем оператор, резольвента которого определяется по формуле (5), будем обозначать через $H_{T}^{0}$.

ЗАмЕчАнИЕ. Выражение (1) используется в физической литературе для символического обозначения целых семейств точечных потенциалов. Соответственно $\lambda_{\mathbf{a}}$ в $(1)$, вообще говоря, не дают корректной параметризации. Математически корректная параметризация точечных возмущений дается теоремой 1. 
Вернемся к $H^{0}$ и $S$, определеным через (2) и (4). Г-поле $\Gamma_{z}$ и $\mathscr{Q}$-функция $Q(z)$ для этой пары строятся следующим образом [9]. Пусть $G^{0}(\mathbf{x}, \mathbf{y} ; z)$ - функция Грина оператоpa $H^{0}$, т.е. интегральное ядро резольвенты $R^{0}(z)$. Известно [7], [10], [11], что $G^{0}(\mathbf{x}, \mathbf{y} ; z)$ раздельно непрерьвна вне диагонали $\mathbf{x}=\mathbf{y}$ при $\nu=2,3$, непрерьвна по совокупности переменных $\mathbf{x}$ и $\mathbf{y}$ при $\nu=1$, а также при каждом $\mathbf{x} \in \mathbb{R}^{\nu}$ выполнено

$$
\int_{\mathbb{R}^{\nu}}\left|G^{0}(\mathbf{x}, \mathbf{y} ; z)\right|^{2} d \mathbf{y}<\infty .
$$

Для каждого $\mathbf{a} \in A$ положим $g_{z}^{\mathbf{a}}=G^{0}(\cdot, \mathbf{a} ; z) ;$ в силу $(6)$ все $g_{z}^{\mathbf{a}}$ лежат в $L^{2}\left(\mathbb{R}^{\nu}\right)$. Пусть теперь $\mathscr{G}=l^{2}(A)$. Тогда операторы $\Gamma_{z}$ задаются по правилу

$$
\Gamma_{z} \xi=\sum_{\mathbf{a} \in A} \xi_{\mathbf{a}} g_{z}^{\mathbf{a}}, \quad \xi=\left(\xi_{\mathbf{a}}\right)_{\mathbf{a} \in A} \in l^{2}(A) .
$$

$\Phi$ ункция $Q(z)$ в стандартном базисе $l^{2}(A)$ задается матрицей

$$
Q_{\mathbf{a b}}(z)=G^{0}(\mathbf{a}, \mathbf{b} ; z)-G^{0}(\mathbf{a}, \mathbf{b} ; \zeta)+C_{\mathbf{a b}}, \quad \mathbf{a}, \mathbf{b} \in A,
$$

где $\left(C_{\mathbf{a b}}\right)$ - матрица ограниченного самосопряженного оператора в $l^{2}(A), \zeta$ - фиксированньй элемент из $\rho\left(H^{0}\right)$.

Мы будем использовать так назьваемую “каноническую” $\mathscr{Q}$-функцию, матрица которой задана следуюшим образом [9]:

$$
Q_{\mathbf{a b}}(z)= \begin{cases}G^{0}(\mathbf{a}, \mathbf{b} ; z) & \text { при } \mathbf{a} \neq \mathbf{b}, \\ G^{0}(\mathbf{a}, \mathbf{a} ; z)-G^{0}(\mathbf{a}, \mathbf{a} ; \zeta) & \text { при } \mathbf{a}=\mathbf{b}\end{cases}
$$

при $\nu=2,3$ и

$$
Q_{\mathbf{a b}}(z)=G^{0}(\mathbf{a}, \mathbf{b} ; z)
$$

при $\nu=1$.

Для удобства ссылок зафиксируем также следующее известное утверждение.

Лемма 1 [9]. Пусть $z \in \rho\left(H^{0}\right), \mathbf{a} \in A, \varphi \in \mathbf{D}\left(H^{0}\right)$. Тогда

$$
\left\langle g_{z}^{\mathbf{a}} \mid\left(H^{0}-\bar{z}\right) \varphi\right\rangle=\varphi(\mathbf{a}) .
$$

Отсюда следует, что при $z, \zeta \in \rho\left(H^{0}\right)$ выполнено включение $g_{z}^{\mathbf{a}}-g_{\zeta}^{\mathbf{a}} \in \mathbf{D}\left(H^{0}\right)$.

2. Локальность квадратичной формы для точечного возмущения в двухи трехмерном случаях. В этом пункте мы будем рассматривать лишь случай $\nu=$ 2,3 . Напомним, что в этом случае функция Грина имеет следующую асимптотику [10]: для любых $\mathbf{y} \in \mathbb{R}^{\nu}$ и $\delta>0$ найдутся функция $\omega \in C^{2}\left(\mathbb{R}^{\nu}\right)$ и число $\alpha \neq 0$ такие, что

$$
G^{0}(\mathbf{x}, \mathbf{y} ; z)= \begin{cases}\omega(\mathbf{x})+\alpha \ln |\mathbf{x}-\mathbf{y}| & \text { при } \nu=2, \\ \omega(\mathbf{x})+\frac{\alpha}{|\mathbf{x}-\mathbf{y}|} & \text { при } \nu=3\end{cases}
$$

при $0<|\mathbf{x}-\mathbf{y}|<\delta$.

Прежде чем перейти к предварительным утверждениям, дадим некоторые пояснения. 
ЗАмЕчаниЕ. Мы отождествляем $l^{2}(A)$ и $L^{2}\left(\mathbb{R}^{\nu}, d \mu\right)$ с мерой $\mu \Omega=\operatorname{card}\{\Omega \cap A\}$; в частности, для $\xi=\left(\xi_{\mathbf{a}}\right)_{\mathbf{a} \in A} \in l^{2}(A)$ имеем $\operatorname{supp} \xi=\left\{\mathbf{a}: \xi_{\mathbf{a}} \neq 0\right\}$.

Лемма 2. Пусть $z \in \rho\left(H^{0}\right), \xi=\left(\xi_{\mathbf{a}}\right) \in l^{2}(A), \Omega$ - открытое мнохсество, содерэащее множество $\operatorname{supp} \xi$ вместе с некоторой его в-окрестностью. Тогда найдется функиия $\varphi$ из $\mathbf{D}\left(H^{0}\right)$ такая, что $\operatorname{supp}\left(\varphi-\Gamma_{z} \xi\right) \subset \Omega$.

ДокАЗАТЕЛЬСТво. Обозначим

$$
\theta=\inf _{\substack{\mathbf{a} \in \operatorname{supp} \\ \mathbf{x} \notin \Omega}}|\mathbf{x}-\mathbf{a}|
$$

по условию $\theta>0$. Выберем числа $d$ и $D$ так, что $0<d<D<\theta$, и функцию $\phi \in C_{0}^{\infty}\left(\mathbb{R}^{\nu}\right)$ такую, что $0 \leqslant|\phi| \leqslant 1, \phi(\mathbf{x})=0$ при $|\mathbf{x}|>D, \phi(\mathbf{x})=1$ при $|\mathbf{x}|<d$. Обозначим $\psi=1-\phi$, $\psi_{\mathbf{a}}=\psi(\cdot-\mathbf{a}), \varphi_{\mathbf{a}}=\psi_{\mathbf{a}} g_{z}^{\mathbf{a}}$. Покажем, что $\varphi_{\mathbf{a}} \in \mathbf{D}\left(H^{0}\right)$.

В смысле обобщенных функций выполнено равенство

$$
\left((i \nabla+\mathbf{A})^{2}+V-z\right) \varphi_{\mathbf{a}}=\psi_{\mathbf{a}}\left((i \nabla+\mathbf{A})^{2}+V-z\right) g_{z}^{\mathbf{a}}-g_{z}^{\mathbf{a}} \Delta \psi_{\mathbf{a}}+2 i \nabla \psi_{\mathbf{a}}(i \nabla+\mathbf{A}) g_{z}^{\mathbf{a}}
$$

Рассмотрим правую часть (9). Первое слагаемое в ней равно нулю в силу равенства (7). Второе слагаемое, очевидно, лежит в $L^{2}\left(\mathbb{R}^{\nu}\right)$. Принадлежность третьего слагаемого к $L^{2}\left(\mathbb{R}^{\nu}\right)$ следует из (8). Таким образом, правая часть (9) является элементом $L^{2}\left(\mathbb{R}^{\nu}\right)$, откуда следует $\varphi_{\mathbf{a}} \in \mathbf{D}\left(H^{0}\right)$, причем правая часть (9) есть выражение для $\left(H^{0}-z\right) \varphi_{\mathbf{a}}$.

В силу $\Lambda$-периодичности $\mathbf{A}$ и $V$ функция $Г$ рина $G^{0}(\mathbf{x}, \mathbf{y} ; z)$ обладает следующим свойством:

$$
G^{0}\left(\mathbf{x}, \mathbf{y}+\mathbf{a}_{i} ; z\right) \equiv G^{0}\left(\mathbf{x}-\mathbf{a}_{i}, \mathbf{y} ; z\right), \quad i \leqslant m,
$$

поэтому существуют постоянные $C_{1}, C_{2}$ такие, что для всех $\mathbf{a} \in A$ вьполнено

$$
\left\|g_{z}^{\mathbf{a}} \Delta \psi_{\mathbf{a}}\right\| \leqslant C_{1}, \quad\left\|\nabla \psi_{\mathbf{a}}(i \nabla+\mathbf{A}) g_{z}^{\mathbf{a}}\right\| \leqslant C_{2}
$$

Обозначим $\varphi=\sum_{\mathbf{a} \in A} \xi_{\mathbf{a}} \varphi_{\mathbf{a}} ;$ как легко видеть, $\varphi \in L^{2}\left(\mathbb{R}^{\nu}\right)$. Кроме того, семейство $\left(g_{z}^{\mathbf{a}} \Delta \psi_{\mathbf{a}}\right) \mathbf{a} \in A$, как и $\left(\nabla \psi_{\mathbf{a}}(i \nabla+\mathbf{A}) g_{z}^{\mathbf{a}}\right) \mathbf{a} \in A$, образует ортогональную систему, поэтому ряд $\sum_{\mathbf{a} \in A} \xi_{\mathbf{a}}\left(H^{0}-z\right) \varphi_{\mathbf{a}}$ сходится в $L^{2}\left(\mathbb{R}^{\nu}\right)$. По теореме о замкнутом графике отсюда следует $\varphi \in \mathbf{D}\left(H^{0}\right)$. В силу построения $\varphi$ вьполнено $\operatorname{supp}\left(\varphi-\Gamma_{z} \xi\right) \subset \Omega$, так что $\varphi-$ требуемая функция. Лемма доказана.

Лемма 3. Имеет место равенство $\mathbf{Q}\left(H^{0}\right) \cap \Gamma_{z} l^{2}(A)=\{0\}$.

ДокАЗАТЕЛЬСтво. Предположим, напротив, что $\varphi \in \mathbf{Q}\left(H^{0}\right) \cap \Gamma_{z} l^{2}(A)$ и $\varphi \neq 0$. Тогда $\varphi=\Gamma_{z} \xi, \xi=\left(\xi_{\mathbf{a}}\right)_{\mathbf{a} \in A} \in l^{2}(A)$ и $\xi_{\mathbf{b}} \neq 0$ при некотором $\mathbf{b} \in A$, и мы можем записать $\xi_{\mathbf{b}} g_{z}^{\mathbf{b}}=\varphi-\Gamma_{z} \eta$, где $\eta=\left(\xi_{\mathbf{a}}-\delta_{\mathbf{a b}} \xi_{\mathbf{b}}\right)_{\mathbf{a} \in A}$. Воспользовавшись леммой 2 , найдем функцию $\phi$, совпадающую в некотором шаре $\Omega$, содержащем точку $\mathbf{b}, \mathrm{c} \Gamma_{z} \eta$. Кроме того, выберем $\omega \in C^{2}\left(\mathbb{R}^{\nu}\right)$ и $\alpha \neq 0$ так, что в $\Omega$ выполнено равенство (8). Тогда на $\Omega$ имеем

$$
-\xi_{\mathbf{b}} \omega+\varphi-\phi= \begin{cases}\alpha \xi_{\mathbf{b}} \ln |\mathbf{x}-\mathbf{y}| & \text { при } \nu=2, \\ \frac{\alpha \xi_{\mathbf{b}}}{|\mathbf{x}-\mathbf{y}|} & \text { при } \nu=3 .\end{cases}
$$


Из (3b) следует, что правая часть последнего равенства лежит в $H_{\text {loc }}^{1}(\Omega)$, для левой же части такое включение не выполнено. Полученное противоречие доказывает лемму.

Теперь наша цель - построить квадратичную форму для $H_{T}^{0}$. Обозначим $\mathbf{D}=\mathbf{Q}\left(H^{0}\right)$ $+\Gamma_{z} l^{2}(A)$ и введем оператор $P: \mathbf{D} \mapsto l^{2}(A)$ соотношением $\varphi-\Gamma_{z} P \varphi \in \mathbf{Q}\left(H^{0}\right)$. Из леммы 1 следует, что $\mathbf{D}$ и $P$ не зависят от выбора $z \in \rho\left(H^{0}\right)$. При построении формы мы ограничимся полуограниченными снизу точечными возмущениями, в связи с чем будем рассматривать лиш те операторы $H_{T}^{0}$, соответствуюшие которым операторы $T$ удовлетворяют следуюшему условию [6], [12], [13]:

при некотором $\zeta<\inf \sigma\left(H^{0}\right)$ оператор $T-Q(\zeta)$ положительно определен.

Обозначим теперь через $h_{z}^{0}, t$ и $t_{z}$ квадратичные формы для операторов $H^{0}-z, T$ и $T-Q(z)$ соответственно.

Лемма 4. При выполнении (11) квадратичная форма $h_{z}^{T}$ для оператора $H_{T}^{0}-z$ замкнута на $\mathbf{Q}\left(H^{0}\right)+\Gamma_{z} \mathbf{Q}(T)$, полуограничена снизу и задается выражением

$$
h_{z}^{T}(\varphi, \varphi)=h_{z}^{0}\left(\varphi-\Gamma_{z} P \varphi, \varphi-\Gamma_{z} P \varphi\right)+t_{z}(P \varphi, P \varphi)
$$

ДокАЗАТЕЛьСТво следует из общих результатов [12] и проводится совершенно аналогично [14] (см. также [15]).

Лемма 5. Для любой функиии ч из $\mathbf{Q}\left(H_{T}^{0}\right)$ выполнено $\operatorname{supp} P \varphi \subset \operatorname{supp} \varphi$.

ДокАЗАТЕЛЬСтво. Пусть, напротив, $\varphi \in \mathbf{Q}\left(H_{T}^{0}\right), \xi=P \varphi$ и $\varphi=0$ в окрестности $\mathbf{b} \in A$, но $\xi_{\mathbf{b}} \neq 0$. Воспользуемся леммой 2 и найдем функцию $\phi \in \mathbf{D}\left(H^{0}\right)$ такую, что $\phi=\Gamma_{z} \xi-\xi_{\mathbf{b}} g_{z}^{\mathbf{b}}$ в некотором шаре $\Omega$, содержащем точку $\mathbf{b}$. Тогда в $\Omega$ должно быть выполнено равенство $\phi=-\xi_{\mathbf{b}} g_{z}^{\mathbf{b}}$. Однако в силу (8) и $(3 \mathrm{~b})$ это равенство не может быть выполнено.

Лемма 6. Пусть $\varphi, \psi \in \mathbf{Q}\left(H_{T}^{0}\right) u \operatorname{supp} \varphi \cap \operatorname{supp} \psi=\varnothing$. Тогда выполнено равенство

$$
h_{z}^{0}\left(\varphi-\Gamma_{z} P \varphi, \psi-\Gamma_{z} P \psi\right)=\langle P \varphi \mid Q(z) P \psi\rangle
$$

ДоКАЗАТЕЛЬСТво во многом следует [6]. Обозначим $\varphi^{0}=\varphi-\Gamma_{z} P \varphi, \psi^{0}=\psi-\Gamma_{z} P \psi$. Из лемм 1 и 5 следует, что для обобщенной функции $\left(H^{0}-z\right) \varphi^{0}$ вьполнено включение

$$
\operatorname{supp}\left(H^{0}-z\right) \varphi^{0} \subset \operatorname{supp} \varphi .
$$

Кроме того, на $\operatorname{supp} \varphi$ выполнено $\psi^{0}=-\Gamma_{z} P \psi$, поэтому

$$
h_{z}^{0}\left(\varphi^{0}, \psi^{0}\right)=\left\langle\left(H^{0}-z\right) \varphi^{0} \mid \psi^{0}\right\rangle=-\left\langle\left(H_{0}-z\right) \varphi^{0} \mid \Gamma_{z} P \psi\right\rangle=-\sum_{\mathbf{a} \in A}(P \psi)_{\mathbf{a}} \overline{\varphi^{0}(\mathbf{a})}
$$


Теперь заметим, что

$$
\varphi(\mathbf{x})=-\sum_{\mathbf{a} \in A}(P \varphi)_{\mathbf{a}} g_{z}^{\mathbf{a}}(\mathbf{x}) \quad \text { при } \mathbf{x} \notin \operatorname{supp} \varphi .
$$

Окончательно, заметив, что в силу леммы $5 \operatorname{supp} P \varphi \cap \operatorname{supp} P \psi=\varnothing$, и подставив (13) в (12), получаем

$$
h_{z}^{0}\left(\varphi^{0}, \psi^{0}\right)=\sum_{\mathbf{a}, \mathbf{b} \in A} \overline{(P \varphi)_{\mathbf{a}}}(P \psi)_{\mathbf{b}} G^{0}(\mathbf{a}, \mathbf{b} ; z)=\langle P \varphi \mid Q(z) P \psi\rangle,
$$

что и требовалось доказать.

Теперь можно доказать основной результат работы.

ТЕорема 2. Для того чтобы оператор $H_{T}^{0}$ был локален в смысле форм, необходимо и достаточно, чтобы оператор $Т$ был такжсе локален в смысле форм.

ДокАЗАТЕльство. Пусть оператор $T$ локален в смысле форм. Возьмем функции $\varphi$, $\psi$ из $\mathbf{Q}\left(H_{T}^{0}\right)$ такие, что $\operatorname{supp} \varphi \cap \operatorname{supp} \psi=\varnothing$. Тогда с применением леммы 6 получаем

$$
h_{z}^{T}(\varphi, \psi)=h_{z}^{0}\left(\varphi-\Gamma_{z} P \varphi, \psi-\Gamma_{z} P \psi\right)+t_{z}(P \varphi, P \psi)=t(P \varphi, P \psi) .
$$

Поскольку $\operatorname{supp} P \varphi \cap \operatorname{supp} P \psi=\varnothing$ в силу леммы 5 , то $t(P \varphi, P \psi)=0$.

Пусть теперь $H_{T}^{0}$ локален в смысле форм. Возьмем $\xi, \eta \in \mathbf{Q}(T)$ такие, что $\operatorname{supp} \xi \cap$ $\operatorname{supp} \eta=\varnothing$. В силу леммы 2 можно найти функции $\varphi, \psi \in \mathbf{D}\left(H^{0}\right)$ такие, что $P \varphi=\xi$, $P \psi=\eta$, причем $\operatorname{supp} \varphi \cap \operatorname{supp} \psi=\varnothing$. Тогда имеем

$$
t(\xi, \eta)=t_{z}(\xi, \eta)+\langle\xi \mid Q(z) \eta\rangle=t_{z}(\xi, \eta)+h_{z}^{0}\left(\varphi-\Gamma_{z} P \xi, \psi-\Gamma_{z} P \eta\right)=h_{z}^{T}(\varphi, \psi)=0 .
$$

Теорема доказана.

В классе операторов $T$, имеющих матрицы в стандартном базисе $l^{2}(A)$, форм-локальность эквивалентна диагональности этой матрицы, поэтому доказанная теорема допускает важный частньй случай.

СлЕДСТВИЕ. В классе операторов $T$, имеющих матричу в стандартном базиcе $l^{2}(A)$, локальным в смысле форм точечным возмущениям $H_{T}^{0}$ соответствуют лишь те операторы, матрица которых диагональна.

3. Замечания. Все утверждения для $\nu=2,3$ сохраняют силу и для оператора Шредингера с однородным магнитным полем [3], т.е. $\mathbf{A}=\mathbf{A}_{u}=\mathbf{B} \times \mathbf{x}$, где $\mathbf{B}$ - постоянньй вектор из $\mathbb{R}^{\nu}$. В этом случае оператор $H^{0}$ уже не является $\Lambda$-периодическим, поэтому его функция Грина не обладает свойством (10). Тем не менее, такой оператор инвариантен относительно действия групшы магнитных трансляций [16], и потому (10) можно заменить на

$$
\begin{aligned}
\left|G^{0}\left(\mathbf{x}, \mathbf{y}+\mathbf{a}_{i} ; z\right)\right| & \equiv\left|G^{0}\left(\mathbf{x}-\mathbf{a}_{i}, \mathbf{y} ; z\right)\right|, \\
\left|(i \nabla+\mathbf{A}(\mathbf{x})) G^{0}\left(\mathbf{x}, \mathbf{y}+\mathbf{a}_{i} ; z\right)\right| & \equiv\left|\left(i \nabla+\mathbf{A}\left(\mathbf{x}-\mathbf{a}_{i}\right)\right) G^{0}\left(\mathbf{x}-\mathbf{a}_{i}, \mathbf{y} ; z\right)\right|, \quad i \leqslant m,
\end{aligned}
$$

что не влияет на дальнейшее изложение. В случаях $H_{0}=-\Delta$ и $H^{0}=\left(i \nabla+\mathbf{A}_{u}\right)^{2}$ все результаты сохраняются и для случая, когда $A$ - прозвольное равномерно дискретное множество [9]. 
4. Одномерный случай. Пусть $\nu=1$ и $H^{0}=-\Delta+V$. Будем рассматривать лишь возмущения с операторами $T$, удовлетворяющими следующему условию: оператор $T$ имеет ограниченный обратньй. Обозначим $B=T^{-1}$.

Лемма 7. Квадратичная форма $h_{z}^{T}$ для $H_{T}^{0}-z$ замкнута на $\mathbf{Q}\left(H^{0}\right)$ и задается выражением

$$
h_{z}^{T}(\varphi, \varphi)=h_{z}^{0}(\varphi, \varphi)+\sum_{\mathbf{a} \in A} B_{\mathbf{a b}} \overline{\varphi(\mathbf{a})} \varphi(\mathbf{b})
$$

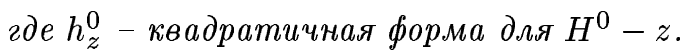

ДокАЗАТЕЛЬСТво повторяет доказательство соответствующего утверждения для $V=0$, приведенное в [1].

Теорема 3. Оператор $H_{T}^{0}$ локален в смысле форм тогда и только тогда, когда оператор $T$ задается в стандартном базисе $l^{2}(A)$ диагональной матричей.

ДокАЗАТЕЛЬСТво. Поскольку $C_{0}^{\infty}(\mathbb{R}) \subset \mathbf{Q}\left(H^{0}\right)$, из $(14)$ сразу следует, что для локальности квадратичной формы точечного возмущения необходимо и достаточно, чтобы оператор $B$ имел диагональную матрицу. Учитьвая связь между $T$ и $B$, получаем утверждение теоремы.

5. Связь с краевыми условиями. Полученные результаты допускают достаточно простое описание на языке граничных условий в точках множества $A$. Для большей наглядности будем считать, что множество $A$ конечно.

Сначала рассмотрим двух- и трехмерный случаи. Как следует из теоремы 1 , любая функция $f$ из области определения любого точечного возмщения $H_{T}^{0}$ вблизи каждой точки $\mathbf{a} \in A$ имеет следующую асимптотику [2]:

$$
f(\mathbf{x})=\phi_{\mathbf{a}}(f)+\psi_{\mathbf{a}}(f) g_{z}^{\mathbf{a}}(\mathbf{x})+o(1), \quad \mathbf{x} \rightarrow \mathbf{a},
$$

где числа $\phi_{\mathbf{a}}(f), \psi_{\mathbf{a}}(f)$ определяются для $f$ единственньм образом. Векторы $\phi(f)=$ $\left(\phi_{\mathbf{a}}(f)\right)_{\mathbf{a} \in A}$ и $\psi(f)=\left(\psi_{\mathbf{a}}(f)\right)_{\mathbf{a} \in A}$ назьваются [13] векторами граничных значений функции $f$. Функция $f$ лежит в $\mathbf{D}\left(H_{T}^{0}\right)$ тогда и только тогда, когда $\phi(f)=C \psi(f)$, где $C$ задается в стандартном базисе $l^{2}(A)$ матрищей $C_{\mathbf{a b}}(z)=T_{\mathbf{a b}}-\delta_{\mathbf{a b}} Q_{\mathbf{a b}}(z)$. Матрица $C$, очевидно, диагональна тогда и только тогда, когда диагональна матрица оператора $T$.

В одномерном случае в качествеграничных значений функции $f$ удобно взять $\phi \mathbf{a}(f)=$ $f^{\prime}(\mathbf{a}+)-f^{\prime}(\mathbf{a}-)$ и $\psi_{\mathbf{a}}(f)=f(\mathbf{a})$; тогда оператору $H_{T}^{0}$ соответствуют краевые условия $\phi(f)=C \psi(f)$ с $C=T^{-1}$. Матрица $C$ также диагональна тогда и только тогда, когда диагональна матрица оператора $T$.

Иными словами, в обоих случаях локальньм в смысле форм точечным возмушениям соотвествуют диагональные матрицы $C$, т.е. в каждом из краевых условий, задающих форм-локальное точечное возмущение, участвуют граничные значения функции, соответствующие одной точке. Соответственно недиагональным матрицам $C$ и, следовательно, краевым условиям, связьвающим граничные значения функции в различны точках, соответствуют нелокальные в смысле квадратичных форм точечные возмущения. 
Автор выражает благодарность В. А. Гейлеру за привлечение внимания к тематике и ценные обсуждения рассмотренных вопросов, а также С. Ю. Доброхотову за постоянный интерес к работе. Автор также признателен рецензенту за полезные замечания.

\section{СПИСОК ЦИТИРОВАННОЙ ЛИТЕРАТУРЫ}

[1] Альбеверио С., Гестези Ф., Хеэг-Крон Р., Хольден Х. Решаемые модели в квантовой механике. М.: Мир, 1991.

[2] Павлов Б.С. Теория расширений и явнорешаемые модели // УМН. 1987. Т. 42. №6. C. $99-131$.

[3] Новиков С. П. Двумерные операторы Шредингера в периодических полях // Современные проблемы математики (Итоги науки и техники ВИНИТИ АН СССР). Т. 23. М.: ВИНИТИ, 1983. C. 3-32.

[4] Albeverio S., Geyler V. A. The band structure of the general periodic Schrödinger operator with point interactions // Comm. Math. Phys. 2000. V. 210. № 1. P. 29-48.

[5] Geyler V.A., Pankrashkin K. V. On fractal structure of the spectrum for periodic point perturbations of the Schrödinger operator with a uniform magnetic field // Operator Theory: Adv. and Appl. 1999. V. 108. P. 259-265.

[6] Шондин Ю. Г. Полуограниченные локальные гамильтонианы для возмущений лапласиана на кривых с угловыми точками в $\mathbb{R}^{4} / /$ ТМФ. 1996. Т. 106. № 2. С. 179-199.

[7] Simon B. Schrödinger semigroups // Bull. Amer. Math. Soc. 1982. V. 7. № 3. P. 447-526.

[8] Крейн М. Г., Лангер Г. К. О дефектных подпространствах и обобщенных резольвентах эрмитова оператора в пространстве $\Pi_{\kappa} / /$ Функцион. анализ и его прилож. 1971. V. 5.№ 2. P. 59-71; № 3. P. 54-69.

[9] Гейлер В.А., Маргулис В. А., Чучаев И.И. Потенциалы нулевого радиуса и операторы Карлемана // Сиб. матем. ж. 1995. Т. 36. № 4. С. 628-641.

[10] Берс Л., Джон Ф., Шехтер М. Уравнения с частными производными. М.: Мир, 1966.

[11] Коротков В. Б. Интегральные операторы. Новосибирск: Наука, 1983.

[12] Бирман М.Ш. К теории самосопряженных расширений положительно определенных операторов // Матем. сб. 1956. Т. 38. №4. С. 431-450.

[13] Derkach V. A., Malamud M. M. Generalized resolvents and the boundary value problems for Hermitian operators with gaps // J. Funct. Anal. 1991. V. 95. № 1. P. 1-95.

[14] Teta A. Quadratic forms for singular pertubations of the Laplacian // Publ. RIMS Kyoto Univ. 1990. V. 26. P. 803-817.

[15] Кошманенко В. Д. Сингулярные билинейные формы в теории возмушений самосопряженных операторов. Киев: Наукова думка, 1993.

[16] Гейлер В. А. Двумерньй оператор Шредингера с однородньм магнитньм полем и его возмущения периодическими потенциалами нулевого радиуса // Алгебра и анализ. 1991. Т. 3. № 3. С. 1-48.

Институт проблем механики РАН, г. Москва

Поступило

E-mail: kpankrashkin@mail.com

25.10 .1999

Исправленньй вариант

15.01 .2001 